()2020. This manuscript version is made available under the CC-BY-NC-ND 4.0 license http:// creativecommons.org/licenses/by-nc-nd/4.0/

\title{
Viscosity effect on a point absorber wave energy converter hydrodynamics validated by simulation and experiment
}

\author{
Siya Jin, Ron J. Patton*, Bingyong Guo \\ School of Engineering and Computer Science, University of Hull, HU6 $7 R X, U K$
}

\begin{abstract}
To achieve optimal power in a wave energy conversion (WEC) system it is necessary to understand the device hydrodynamics. To maximize conversion efficiency the goal is to tune the WEC performance into resonance. The main challenge then to be overcome is the degree to which non-linearity in WEC hydrodynamics should be represented. Although many studies use linear models to describe WEC hydrodynamics, this paper aims to show that the non-linear viscosity should be carefully involved. To achieve this an investigation into the hydrodynamics of a designed 1/50 scale point absorber wave energy converter (PAWEC) in heave motion only is implemented to indicate the non-linear viscosity effect. A non-linear state-space model (NSSM) considering a quadratic viscous term is used to simulate PAWEC behaviors. The non-linear model is compared with the linear counterpart, and validated by computational fluid dynamics (CFD) and experimental data. A conclusion is drawn that the non-linear PAWEC hydrodynamics (including amplitude and phase responses, conversion efficiency) close to resonance or at high wave heights can only be described realistically when the non-linear viscosity is correctly taken into account. Inaccuracies in its representation lead to significant errors in the tuning procedure which over-predict the dynamic responses and weaken the control system performance.
\end{abstract}

\footnotetext{
*Corresponding author

Email addresses: siya_jin@126.com (Siya Jin), r.j.patton@hull.ac.uk (Ron J. Patton), B.Guo@exeter.ac.uk (Bingyong Guo)
} 
Keywords: Wave energy conversion, Viscosity influence, State-space model, Three-dimensional CFD, Physical experiment

3

4

6 detailed in [3, 4, 5]. In the process of studying a complete WEC system, it is

7 of fundamental importance to obtain an overall and applicable hydrodynamic

8 description for the way in which the device interacts with incident waves. This

- mathematical description is important for suggesting the power take-off (PTO)

10 design as well as the control system development since these WEC subsystems

11 are influenced by the dynamic interaction that the WEC device has with the 12 wave motion [6, 7, 8, 9].

A variety of methods have been developed to describe WEC hydrodynamics [10], the most widely adopted of which is the conventional linear modeling method derived from the boundary element method (BEM) based on the linear potential flow theory. This approach has the advantages of: (i) providing convenient hydrodynamic predictions for a given WEC device in both the frequency and the time domains [11, 12]; (ii) easing the integration with control method as a hydrodynamic plant [9, 13, 14]. Nevertheless, this method may over-predict the WEC motion and power production, especially at the most promising conditions, such as resonance and high wave heights [8, 15]. This can be attributed to the linear assumptions accompanying this method [16, 17, such as (i) the wave should be linear; (ii) the WEC motion should be small; (iii) the WEC effective dimension should be comparable with the incoming wave length. In this case, the practical non-linear dissipative factors (e.g., large wave height, viscosity, slamming, over-topping, etc.) are ignored.

Some investigators prefer to conduct physical experiments [18, 19] or imple- 
57 a non-linear dynamic model to perform WEC behaviors.

ment computational fluid dynamics (CFD) simulations by solving the NavierStokes equations directly. These approaches naturally take appropriate nonlinear WEC performances into account. For example, through CFD analysis, (i) Yu et al. [20] demonstrated that the over-topping phenomenon reduced the amplitude response of a two-body floating point absorber system; (ii) Wei et al. 21] concluded that the viscosity influence on the bottom hinged Oscillating Wave Surge Converter was relevant to the flap width. However, these approaches are complex and not straightforward for control application.

Thus, the requirement for improved mathematical models involving nonlinear factors is increasing, especially as advanced control application is one of the main goals. One method is to approximate the non-linear effect by a linear equivalent term. For instance, Son et al. [22] applied a linear equivalent viscous damping term into the conventional linear model to represent the viscous effect. From free decay studies in a CFD wave tank, Davidson et al. 23] summarized the variation of the linearized radiation and added mass terms against the initial position. Verified by experimental results in [24], a numerical dynamic model supplied with a linearization of the quadratic viscous force was valid to perform the dynamics of the self-reacting PAWEC under small wave conditions with low body velocity. However, this approach is limited, as the linearized terms are required to be adjusted with varying test condition. Therefore, the inclusion of practical non-linear terms is expected. As suggested by Beatty [24], it is necessary to improve the accuracy of the dynamic model with a quadratic viscous drag under larger waves and/or higher body velocities. Comparing with CFD data, Bhinder et al. 25] showed that the conventional linear model together with additional quadratic viscous term offers an improvement in describing the surging floating WEC performance. From experimental free decay studies, Guo et al. 26] indicated that a model including non-linear viscous and frictional terms can be more practical in representing the non-linear behaviors under different initial displacements. These studies highlight the necessity of achieving Inspired by the above background, a study regarding a designed $1 / 50$ scale 


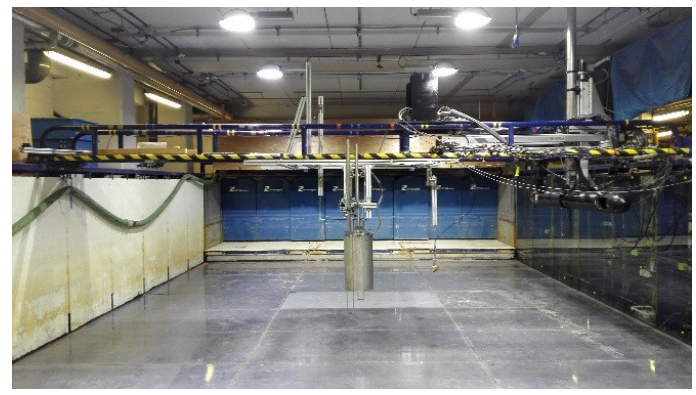

Figure 1: University of Hull PAWEC experimental wave tank.

59 vertical oscillating PAWEC device (Fig. 1) has been ongoing at University of 6 Hull [27, 28. The aim of this paper is to explore and gain further knowledge of 61 the viscosity effect on the designed PAWEC dynamic behavior, and thereby to 62 design an applicable non-linear state-space model (NSSM) considering viscosity.

${ }_{63}$ The main contributions of this paper are as follows:

64

- The variation of the PAWEC amplitude and phase responses versus wave frequency at three kinds of wave heights (small, moderate and high) were summarised via LSSM (linear state-space model), NSSM, CFD and experiment. These tests clearly show the substantial discrepancies of the predicted results between the non-linear (including NSSM, CFD and and experiment in this work) and linear methods. The non-negligible viscosity effect on wave-PAWEC interaction around resonance or at high wave heights has been discussed. It shows that the non-linear viscous damping is significantly important at large oscillations. Thus it would be necessary to apply a NSSM into control system development for achieving optimal power conversion efficiency.

- Although the rule of power conversion efficiency has been established in [29], few works summarise the non-linear characteristics of this factor. In this study, the PAWEC power conversion efficiencies have been summarised versus wave frequency, PTO damping coefficient at three wave heights via LSSM and NSSM. The results indicate that the power conver- 
sion efficiency has clear non-linearity against wave height. More importantly, the optimal PTO damping or wave condition can be incorrectly predicted by the LSSM so that this approach loses ability in predicting maximum efficiency. This implies that the LSSM would mislead not only the selection of an optimal PTO system but also the control design.

The paper is organized as follows. Section 2 outlines the materials and methods employed in this work, i.e., LSSM, NSSM, CFD, the experimental testing platform and the illustrative case studies. Results and discussions related to the case studies are drawn in Section 3 . Section 4 concludes the study.

\section{Materials and methods}

The adopted materials and methods for studying the viscosity effect on the PAWEC hydrodynamics are outlined in this section. The conventional LSSM is derived to represent the PAWEC motion by approximating the radiation force with a 4-order system, described in Section 2.1. Taking a quadratic viscous term into account, the NSSM is designed in Section 2.2. Sections 2.3 and 2.4 describe the CFD and experimental platforms, respectively. The representative case studies implemented in LSSM, NSSM, CFD and experiments are illustrated in Section 2.5

\subsection{The conventional LSSM}

\subsubsection{Hydrodynamic descriptions in time and frequency domains}

The widely used time domain WEC hydrodynamic model from [30] can be expressed as:

$$
\left(M+m_{\infty}\right) \ddot{z}(t)+\int_{0}^{t} k_{r}(t-\tau) \dot{z}(\tau) d \tau+K z(t)=f_{e}(t),
$$

where $M$ represents the body mass; $f_{e}(t)$ is the excitation force due to the incident wave; $m_{\infty}, k_{r}(t)$ are the frequency dependent added mass at the infinite frequency and the radiation force Impulse Response Function (IRF); $K$ and $z(t)$ are the hydrostatic stiffness and the vertical displacement, respectively. 
In this work, only the regular wave is studied, described as:

$$
\lambda(t)=A_{\text {wave }} \cos (\omega t)=\Re\left\{A_{\text {wave }} e^{j \omega t}\right\},
$$

where $\lambda(t), A_{\text {wave }}, \omega$ are the incident wave elevation, amplitude and frequency, respectively; $\Re$ represents the real part of a complex number.

Considering the linear theory, the $f_{e}(t)$ amplitude is proportional to that of the incident wave:

$$
f_{e}(t)=A_{\text {wave }} F_{e c}(\omega) \cos (\omega t+\varphi(\omega))=A_{\text {wave }} \Re\left\{\hat{F}_{e c} e^{j \omega t}\right\},
$$

where $\hat{F}_{e c}$ is the complex excitation force coefficient in the frequency domain. $\hat{F}_{e c}=F_{e c}(\omega) e^{j \varphi(\omega)}$, where $F_{e c}(\omega)$ and $\varphi(\omega)$ are the corresponding modulus and phase angle, respectively.

In Eq. (1), the summation of the infinite-frequency added mass inertial force and the inviscid hydrodynamic damping force represents the radiation force $f_{r}(t)$, corresponding to the hydrodynamic reaction caused by the WEC oscillation against the neighbour flow:

$$
f_{r}(t)=m_{\infty} \ddot{z}(t)+\int_{0}^{t} k_{r}(t-\tau) \dot{z}(\tau) d \tau .
$$

Ogilvie [31] rewrote Eq. (11) into the frequency domain as:

$$
\left\{-[M+m(\omega)] \omega^{2}+K+j \omega B(\omega)\right\} Z(j \omega)=A_{\text {wave }} \hat{F}_{e c},
$$

where $m(\omega)$ is the added mass (substitute $M_{t}(\omega)$ for $\left.M+m(\omega)\right) ; Z(j \omega), B(\omega)$ are the WEC displacement, inviscid radiation damping coefficient in the frequency domain. Ogilvie [31] also established the relationship between $B(\omega)$ and $k_{r}(t)$ as:

$$
B(\omega)=\int_{0}^{\infty} k_{r}(t) \cos (\omega t) d t
$$

Hence,

$$
k_{r}(t)=(2 / \pi) \int_{0}^{\infty} B(\omega) \cos (\omega t) d \omega .
$$

Transforming Eq. (5), the WEC velocity $\hat{V}=j \omega Z(j \omega)$ is obtained:

$$
\hat{V}=j \omega Z(j \omega)=\frac{A_{w a v e} \hat{F}_{e c}}{j\left[\omega M_{t}(\omega)-K / \omega\right]+B(\omega)},
$$


Note that the so-called resonance is obtained at $\omega=\omega_{0}=\left[K / M_{t}\left(\omega_{0}\right)\right]^{1 / 2}\left(\omega_{0}\right.$ is the undamped natural frequency) with the vanishing imaginary part. At resonance, it is noticeable that (i) the WEC velocity is in phase with the wave excitation force; (ii) the WEC velocity magnitude would reach its maximum if both $\hat{F}_{e c}$ and $B(\omega)$ have negligible variations with $\omega$.

Transforming Eq. (8), the response amplitude operator (RAO) is obtained:

$$
\mathrm{RAO}=\frac{|Z(j \omega)|}{A_{\text {wave }}}=\frac{F_{e c}(\omega)}{\left|-\omega^{2} M_{t}(\omega)+K+j \omega B(\omega)\right|},
$$

Note that the variation against $\omega$ facilitates a determination of the maximum RAO value at $\omega=\omega_{0}^{\prime}=\left[\omega_{0}^{2}-B_{\omega_{0}^{\prime}}^{2} / 2 M_{t}\left(\omega_{0}^{\prime}\right)^{2}\right]^{1 / 2}$, by assuming both $F_{e c}(\omega)$ and $B(\omega)$ have indistinctive variations with $\omega$. Clearly, $\omega_{0}^{\prime}$ is lower than $\omega_{0}$ due to the damping term $B_{\omega_{0}^{\prime}}^{2} / 2 M_{t}\left(\omega_{0}^{\prime}\right)^{2}[17$.

In the linear potential flow theory, firstly, the hydrodynamic damping only considers the radiation damping $B(\omega)$ by excluding the non-linear dissipative terms. Compared to non-linear damping effects, radiation damping is negligible, as discussed in $[22,24]$. Secondly, the $\hat{F}_{e c}$ is almost in phase with the incident wave at low wave frequencies. Thus combining Eqs. (8) and (9), when a WEC reaches its resonance, the following optimal WEC performance criteria can be achieved together: (i) $\omega_{0}^{\prime}$ has little or no difference relative to $\omega_{0}$; (ii) both the RAO and velocity values reach the maximum; (iii) the WEC velocity is in phase with the excitation force; (iv) the WEC motion is shifted by approximately $90^{\circ}$ relative to the regular wave motion; (v) the WEC power reaches its maximum. This paper will discuss whether or not all of these optimal criteria are still valid at the so-called resonance $\left(\omega=\omega_{0}\right)$ with the consideration of practical non-linear factors, as shown in Sections 3.2 and 3.3 .

\subsubsection{Convolution approximation of the radiation force}

To avoid the complex calculation and inconvenient application for control strategy resulting from the convolution term in Eq. (1) in the time domain, the following state-space model is identified to approximate the convolution 
operation:

$$
\begin{aligned}
\dot{\mathbf{X}}_{\mathbf{r}}(t) & =\mathbf{A}_{\mathbf{r}} \mathbf{X}_{\mathbf{r}}(t)+\mathbf{B}_{\mathbf{r}} \dot{z}(t), \\
f_{r}^{\prime}(t) & =\mathbf{C}_{\mathbf{r}} \mathbf{X}_{\mathbf{r}}(t) \approx \int_{0}^{t} k_{r}(t-\tau) \dot{z}(\tau) d \tau,
\end{aligned}
$$

where $\mathbf{X}_{\mathbf{r}} \in \mathbb{R}^{m \times 1}$ is the state vector of the identified system; $\mathbf{A}_{\mathbf{r}} \in \mathbb{R}^{m \times m}, \mathbf{B}_{\mathbf{r}} \in$ $\mathbb{R}^{m \times 1}$ and $\mathbf{C}_{\mathbf{r}} \in \mathbb{R}^{1 \times m}$ are system matrices, respectively. Various identification methods of the state-space model were described in [12]. This paper make use of the realization theory, implemented via the imp2ss command combined with the order reduction function balmar in MATLAB ${ }^{\circledR}$.

\subsubsection{LSSM for the designed PAWEC}

The designed PAWEC is a cylindrical floater with $500 \mathrm{~kg} / \mathrm{m}^{3}$ in density, $0.3 \mathrm{~m}$ in diameter and $0.28 \mathrm{~m}$ in draught. Based on these physical properties, the corresponding frequency dependent hydrodynamic parameters such as $m_{\infty}, m(\omega), B(\omega)$, RAO and $\hat{F}_{e c}$ can be calculated through the BEM software ANSYS/AQWA (see Figs. 2a and 33). As observed, when the incident wave frequency corresponds to the PAWEC natural frequency $(5.14 \mathrm{rad} / \mathrm{s})$, the motion reaches its maximum and has nearly $90^{\circ}$ phase lag relative to the incident wave. This coincides with the resonance phenomena mentioned in Section 2.1.1.

Referring to the achieved hydrodynamic parameters, whilst considering the trade-offs in accuracy and complexity, a 4-order state-space model has been identified to approximate the convolution term based on Eqs. (7) and (10), as shown in Fig. 2b. The related system matrices are:

$$
\begin{aligned}
& \mathbf{A}_{\mathbf{r}}=\left[\begin{array}{cccc}
-2.9050 & -4.3129 & 3.1027 & -1.0862 \\
4.3129 & -0.0142 & 0.1668 & -0.0881 \\
-3.1027 & 0.1668 & -4.1044 & 5.2748 \\
-1.0862 & 0.0881 & -5.2748 & -2.2996
\end{array}\right], \\
& \mathbf{B}_{\mathbf{r}}=\left[\begin{array}{llll}
-3.9615 & 0.2639 & -1.8048 & -0.7765
\end{array}\right]^{T}, \\
& \mathbf{C}_{\mathbf{r}}=\left[\begin{array}{llll}
-3.9615 & -0.2639 & 1.8048 & -0.7765
\end{array}\right] .
\end{aligned}
$$

${ }_{82}$ Then replacing the convolution term in Eq. (1) by Eq. (10), the PAWEC LSSM 


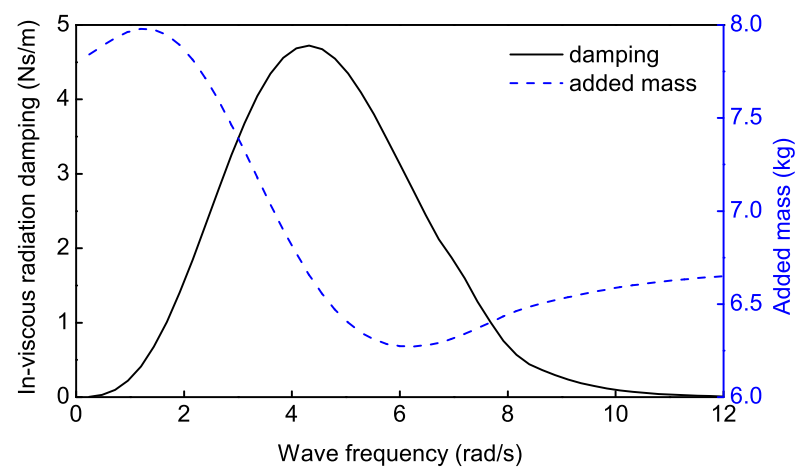

(a)

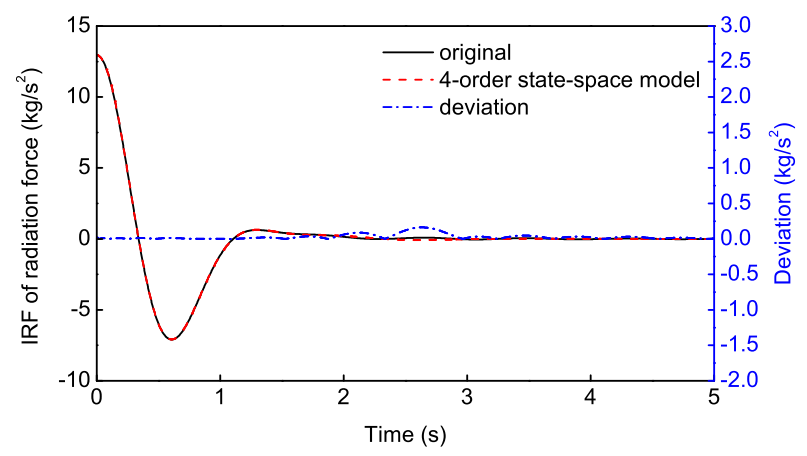

(b)

Figure 2: Radiation force parameters of the PAWEC obtained via ANSYS/AQWA. (a) Added mass and inviscid radiation damping coefficient. (b) Comparison of the $k_{r}(t)$ for the original and estimated results obtained via Eq. 7 and the identified 4-order state-space model, respectively.

${ }_{183}$ is achieved:

184

$$
\begin{aligned}
\dot{\mathbf{X}}(t) & =\mathbf{A X}(t)+\mathbf{B} f_{e}(t), \\
z(t) & =\mathbf{C X}(t),
\end{aligned}
$$




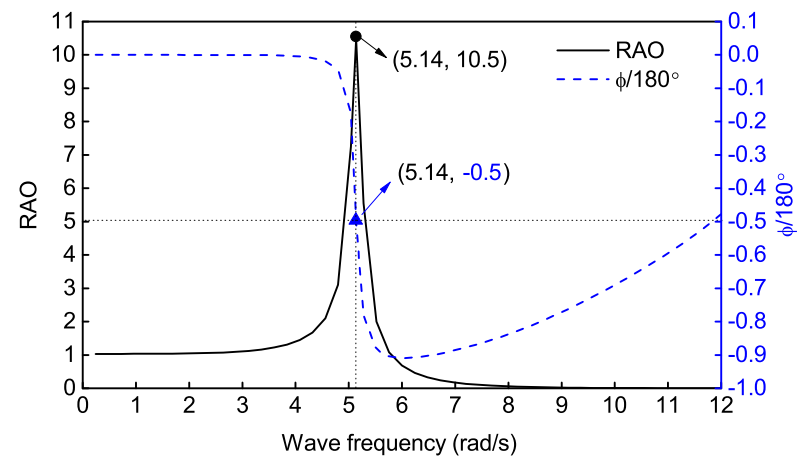

(a)

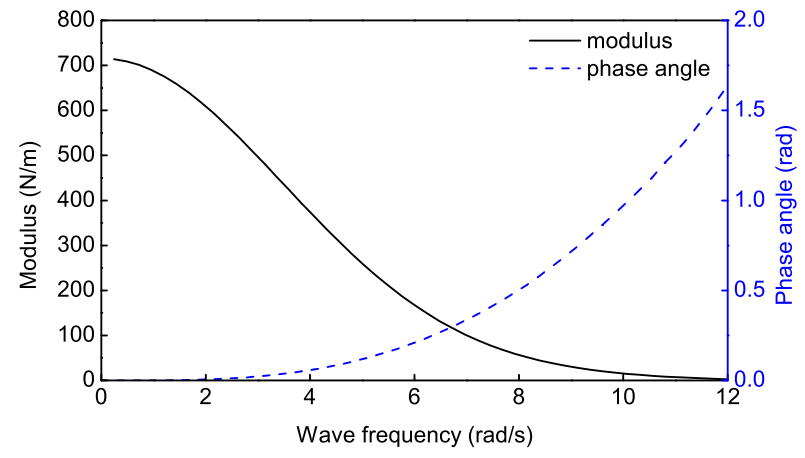

(b)

Figure 3: Hydrodynamic parameters of the PAWEC obtained through ANSYS/AQWA. (a) RAO and phase shift $\phi$ relative to the incident wave motion. (b) Modulus and phase angle of $\hat{F}_{e c}$.

${ }_{185}$ where $\mathbf{X}=\left[\begin{array}{lll}\mathbf{X}_{\mathbf{r}}(t) & z(t) & \dot{z}(t)\end{array}\right]^{T}$; the system matrices are:

186

$$
\begin{aligned}
\mathbf{A} & =\left[\begin{array}{ccc}
\mathbf{A}_{\mathbf{r}} & \mathbf{0}_{\mathbf{4} \times \mathbf{1}} & \mathbf{B}_{\mathbf{r}} \\
\mathbf{0}_{\mathbf{1} \times \mathbf{4}} & 0 & 1 \\
-\mathbf{C}_{\mathbf{r}} / M_{t} & -K / M_{t} & 0
\end{array}\right], \\
\mathbf{B} & =\left[\begin{array}{lll}
\mathbf{0}_{\mathbf{1} \times \mathbf{4}} & 0 & 1 / M_{t}
\end{array}\right]^{T} \\
\mathbf{C} & =\left[\begin{array}{lll}
\mathbf{0}_{\mathbf{1} \times \mathbf{4}} & 1 & 0
\end{array}\right]
\end{aligned}
$$

187

188

189

\subsection{Proposed NSSM for the designed PAWEC}

As described in [16, LSSM may not be applicable for describing the hydrodynamics of a slender structure satisfying: effective diamter/wave length < 
0.2. The dominant frequency for achieving efficient PAWEC oscillation varies in the range: $\omega \leq 6.24 \mathrm{rad} / \mathrm{s}$ (see Fig. 3a). According to $\lambda \approx 2 \pi g / \omega^{2}$ [32], the lower bound of the wave length applied to the PAWEC approximates $1.5 \mathrm{~m}$. This shows that the designed PAWEC with effective diameter of $0.3 \mathrm{~m}$ should be regarded as a slender structure. Under this situation, the viscosity term is essential and must be included in the PAWEC hydrodynamic model description. Hence, the quadratic viscous term in the Morison equation [33] is considered as:

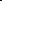

where $f_{v}(t)$ is the viscous force; $r$ is the PAWEC radius; $u(t)$ is the flow vertical velocity, approximate to $\omega A_{\text {wave }} \sin (\omega t) ; C_{d}$ is the viscous coefficient, an empirical value generally predicted through Experimental/CFD test. In this work, the PAWEC $C_{d}$ was predicted via CFD simulation and validated by experimental data described in Section 3.1

Superimposing the quadratic viscous force into Eq. [12, the NSSM is constructed:

$$
\begin{aligned}
\dot{\mathbf{X}}(t) & =\mathbf{A X}(t)+\mathbf{B} f_{e}(t)+\mathbf{B} f_{v}(t), \\
z(t) & =\mathbf{C X}(t) .
\end{aligned}
$$

Referring to Eq. (9), the non-linear RAO considering viscosity can now be considered equivalent to a linear form:

$$
\mathrm{RAO}=\frac{|Z(j \omega)|}{A_{\text {wave }}}=\frac{F_{e c}(\omega)}{\left|-\omega^{2} M_{t}(\omega)+K+j \omega B_{\text {hyd }}\right|} .
$$

where $B_{h y d}$ is the total hydrodynamic damping coefficient including inviscid and viscous components: $B_{h y d}=B(\omega)+B_{\text {vis }}$. Note that: through Eq. 14, the magnitudes of viscous force $f_{v}(t)$ and the related viscous damping coefficient $B_{v i s}$ highly depend upon the relative velocity $v_{r}$ between the wave and the floater. This indicates that a higher $v_{r}$ corresponds to a larger $B_{v i s}$. Besides, it is well known that the $v_{r}$ value is associated with both the wave frequency $\omega$ and the wave height $H$. Therefore, in the non-linear model, both $\omega$ and $H$ would be the variable parameters for $B_{v i s}$ and $B_{h y d}$, described as $B_{v i s}(\omega, H)$ and $B_{h y d}(\omega, H)$, respectively. This is clearly distinguished from the frequency 
dependent $B_{\text {hyd }}(\omega)$ (corresponding to $B(\omega)$ described in Fig. 2a) for the linear theory. This implies that the non-linearities of the hydrodynamic responses under varied wave heights are significant, as discussed in Section 3.2 .

Recall the NSSM in Eq. (15), the remaining uncertain parameter is $C_{d}$. To determine $C_{d}$, the least-squares technique is applied by comparing the NSSM result with CFD output:

$$
p_{e}=\min _{\mathrm{p}} \sum_{\mathrm{i}}\left(z_{N S S M}\left(t_{i}, p\right)-z_{C F D}\left(t_{i}\right)\right)^{2},
$$

where $z_{N S S M}\left(t_{i}, p\right)$ is obtained by solving Eq. 15 via ODE solver in MATLAB ${ }^{\circledR}$; $z_{C F D}\left(t_{i}\right)$ is extracted from the CFD simulation; $p$ and $p_{e}$ represent the uncertain parameter and the estimated parameter with the best fitting, respectively.

\subsection{CFD testing platform}

To thoroughly demonstrate the viscosity effect on wave-PAWEC interaction, numerical simulations in the CFD package ANSYS/LS-DYNA 34 were performed. The CFD testing platform mainly consists of: (i) generating stable wave (Section 2.3.2); (ii) conducting efficient wave-PAWEC interaction reproduction (Section 2.3.3).

\subsubsection{Fundamental CFD theory}

The flow model represented in ANSYS/LS-DYNA solved by the compressible Navier-Stokes equations together with the continuity equation, in contrast to the inviscid, irrotational and incompressible fluid model applied in the linear potential flow theory (Sections 2.1 and 2.2):

$$
\begin{aligned}
& \frac{\partial \vec{v}}{\partial t}+\vec{v} \cdot \nabla \vec{v}=-\frac{1}{\rho} \nabla P+\nu \nabla^{2} \vec{v}+\frac{1}{3} \nu \nabla(\nabla \cdot \vec{v})+\vec{g}, \\
& \frac{\partial \rho}{\partial t}+\rho \nabla \cdot \vec{v}=0,
\end{aligned}
$$

where $\vec{v}, P$ and $\nu$ are the fluid velocity, pressure and kinematic viscosity, respectively; $\vec{g}$ is the external acceleration applied to the fluid (in this work, it represents the gravity acceleration). Clearly, the fluid viscosity effect has been taken into account through Eq. (18). 




Figure 4: Numerical wave tank setup in ANSYS/LS-DYNA.

\subsubsection{Wave generation}

Considering the trade-off between generating stable wave and efficient computation, several techniques were employed while constructing the numerical wave tank (NWT). (i) Since the model is symmetrical, a half model was simulated along the symmetrical plane. (ii) To avoid the unnecessary wave-structure interaction introduced by the wave-maker, a nodes-layer with prescribed displacement in the inflow boundary was introduced for substitute. (iii) To reduce the wave reflection and standing wave, a ramp connecting with a sponge area in the downstream was built to dissipate the propagating energy. According to the paddle wave-maker theory [35, 36, the regular wave is generated:

$$
\begin{aligned}
\frac{H}{S} & =\frac{4 \sinh k_{0} h}{k_{0} h} \frac{k_{0} h \sinh k_{0} h-\cosh k_{0} h+1}{\sinh 2 k_{0} h+2 k_{0} h}, \\
\Delta \theta & =\arctan \left(\frac{S}{2 h}\right), \\
\theta(t) & =\Delta \theta \sin (\omega t)
\end{aligned}
$$

where $H$ is the objective wave height; $h$ is the water depth; $S$ is the wavemaker stroke; $k_{0}$ is the wave number depending upon $\omega^{2}=g k_{0} \tanh k_{0} h ; \Delta \theta$ is the wave-maker swing angle amplitude; $\theta(t)$ is the wave-maker displacement. Consequently, as demonstrated in Fig. 4. a NWT $13 \mathrm{~m}$ in length, $0.75 \mathrm{~m}$ in width, and filled with $0.55 \mathrm{~m}$ depth of water, $0.7 \mathrm{~m}$ depth of air was constructed.

Fig. 5 shows a generated wave at $H=0.08 \mathrm{~m}$ and $\omega=3.9 \mathrm{rad} / \mathrm{s}$. As observed, the obtained numerical wave height is nearly $0.073 \mathrm{~m}$, which shows good 


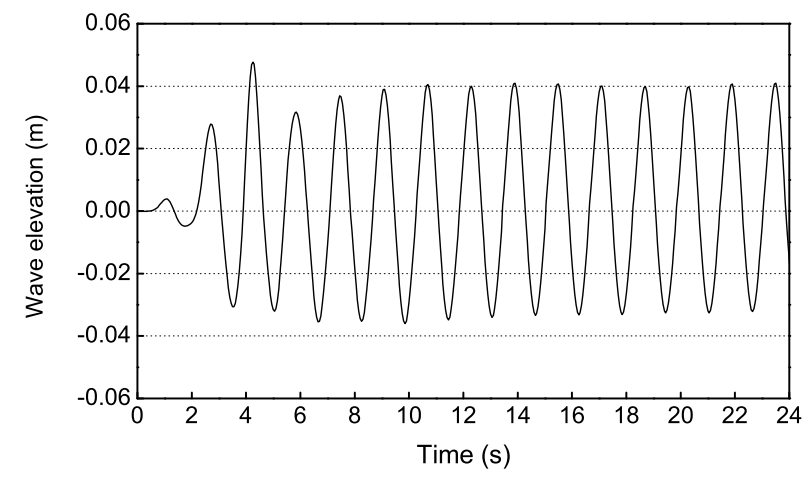

Figure 5: Wave elevation history generated in the NWT at $H=0.08 \mathrm{~m}$ and $\omega=3.9 \mathrm{rad} / \mathrm{s}$.

263 agreement with the objective value. This suggests the feasibility of ANSYS/LS-

${ }_{264}$ DYNA in generating waves. Note that: the objective wave height of $0.08 \mathrm{~m}$ is

265 the experimental wave condition. Hence, a numerical wave height of $0.073 \mathrm{~m}$ in

${ }_{266}$ the NWT is obtained to approximate the experimental condition of $0.08 \mathrm{~m}$ in

267 this work.

\subsubsection{Wave-PAWEC interaction}

In the process of calculating the floater hydrodynamic performance through CFD, it is essential to obtain accurate pressure on the wetted surface. This is highly dependent on the grid quality. Hydrostatic pressure testing was implemented to testify the grids convergence, by pushing the PAWEC bottom surface gradually to $0.28 \mathrm{~m}$ beneath the water surface in the NWT. When the grid sizes were reduced to $0.01 \mathrm{~m}, 0.16 \mathrm{~m}$ and $0.3 \mathrm{~m}$ in the interaction zone, inflow boundary and back wall of the tank, respectively (detailed in Fig. 4), the simulated hydrostatic pressure of the PAWEC bottom surface converged to the theoretical value of $2744 \mathrm{~Pa}$ at $0.28 \mathrm{~m}$ underwater (see Fig. 6). Therefore, this grids solution was adopted in this work.

\subsection{Physical experimental testing platform}

The physical experiments were carried out in the Hull University Total Environment Simulator Wave Tank shown in Fig. 11 The physical tests were 


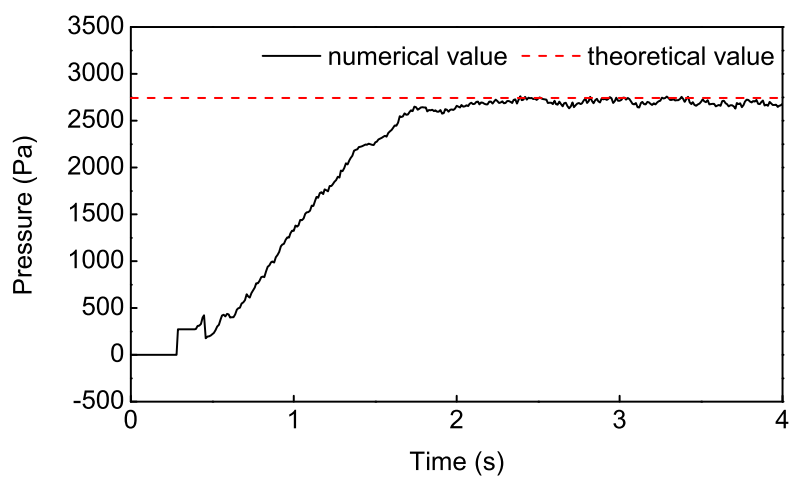

Figure 6: The PAWEC's bottom hydrostatic pressure history while moving from $0.02 \mathrm{~m}$ above to $0.28 \mathrm{~m}$ beneath the water surface.

employed to validate the LSSM, NSSM and CFD approaches. The testing platform is detailed in Fig. 7. A linear variable displacement transducer (LVDT), an accelerometer (Accel) and 5 pressure sensors (PSs) were used to measure the PAWEC displacement, acceleration and bottom hydrodynamic pressure, respectively. The wave elevation was monitored by the wave gauges (WGs). Additionally, roller bearings were used between the vertical guide-bar and the gantry to reduce the contact friction from PAWEC oscillation. However, through the experimental data (see Figs. 8 and 12), there still exists a slight mechanical friction which impedes the PAWEC motion. The mechanical friction effect was discussed in [26], which will not be further described.

\subsection{Case studies}

This section details the three illustrative case studies (free decay motion, forced oscillation and power conversion efficiency tests) implemented in LSSM, NSSM, CFD and the experimental platform, respectively (with corresponding tests results detailed in Section 3). The related parameters are given in Table 1.

Case 1 - free decay motion testing: The PAWEC was released from a nonzero initial position away from its equilibrium where the motion then decayed to the equilibrium. This test was conducted to determine the unknown $C_{d}$ in 


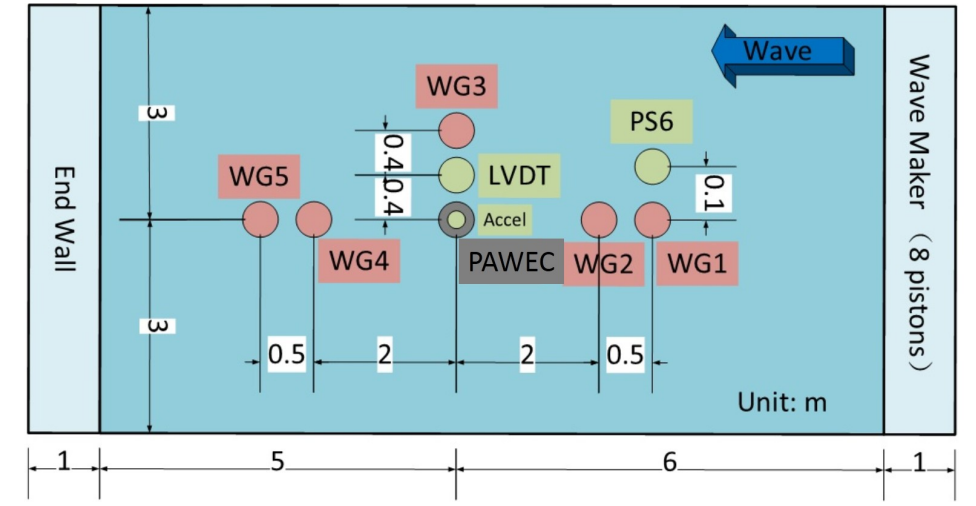

(a)

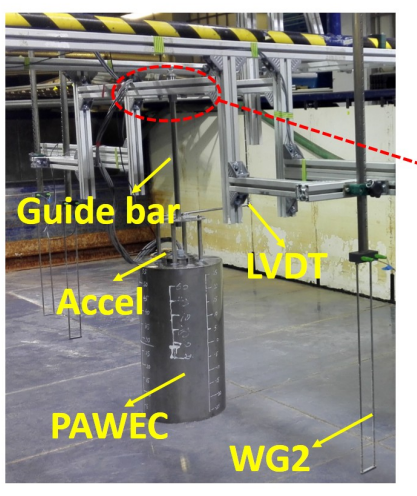

(b)

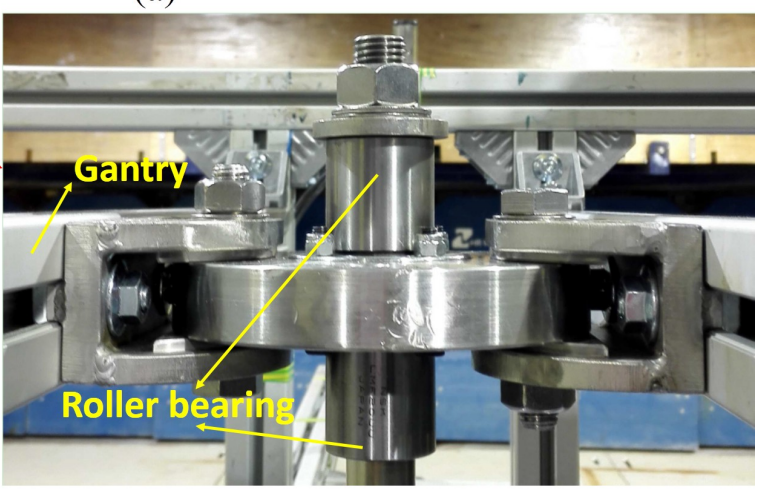

(c)

Figure 7: (a) Scenario of the experimental wave tank. (b) Close-up of the experimental set-up. (c) Close-up of the connections.

the NSSM, by comparing the achieved results from the NSSM with the CFD output, based on Eq. (17). Moreover, physical test data were offered to evaluate the predicted $C_{d}$.

Case 2 - forced oscillation testing: The PAWEC was excited by the regular waves with various wave frequencies at three wave heights. The tests were carried out to state the superiority of the NSSM over the LSSM in representing the wave-PAWEC interaction at various wave conditions. More importantly, the viscosity influence on the PAWEC performance regarding amplitude and phase responses would be discussed. The three adopted wave heights (shown in Table 


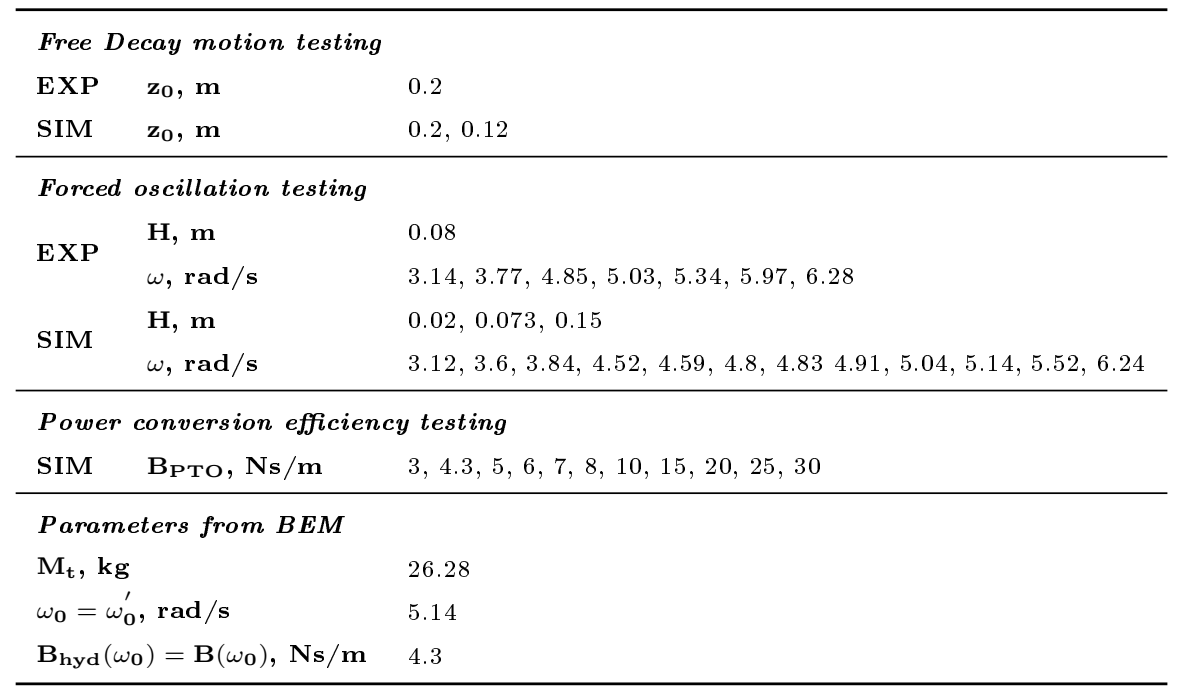

Table 1: Related parameters used in the case studies. $z_{0}$ represents the non-zero initial released displacement against the equilibrium. Abbreviation: $\mathrm{EXP}=$ Experiment, SIM $=$ LSSM/NSSM/CFD.

1) correspond to small, moderate and high wave states in practice 37 .

Case 3 - power conversion efficiency testing: The PAWEC power conversion efficiency variation against wave condition was predicted by introducing a linear PTO into the LSSM and NSSM. Simplifying the PTO as a linear damper and superposing it into Eq. (15), the PAWEC power conversion efficiency could be calculated as 32 :

$$
\begin{gathered}
\bar{P}=\frac{1}{T} \int_{0}^{T} B_{P T O} \dot{z}(t)^{2} d t, \\
P_{\text {wave }}=\frac{1}{4 \omega} \rho g^{2} A_{\text {wave }}{ }^{2} D, \\
C=\frac{\bar{P}}{P_{\text {wave }}}
\end{gathered}
$$

where $\bar{P}$ is the average power generated by the PTO; $P_{\text {wave }}$ is the available wave power on the effective floater diameter; $C$ is the PAWEC power conversion efficiency; $B_{P T O}$ is the PTO damping coefficient. The above equations indicate that the power conversion efficiency is dependent on both the WEC hydrodynamic performance and the employed PTO damping. It is well known that the maximum conversion efficiency is achieved at the WEC natural frequency when 
$B_{P T O}=B_{\text {hyd }}[29]$. In the linear model, the optimal PTO damping coefficient is $4.3 \mathrm{Ns} / \mathrm{m}$ at resonance for the designed PAWEC (see Fig. 2a).

\section{Results and discussions}

This section demonstrates the corresponding results for the three case studies described in Section 2.5. The determination of the uncertain parameter $C_{d}$ is given in Section 3.1. The viscosity effect on the PAWEC amplitude and phase responses, as well as the power conversion efficiency are detailed in Sections 3.2 and 3.3 respectively.

\subsection{Identification of the unknown parameters in NSSM}

According to Section 2.2 the remaining unknown parameter in the NSSM for the designed PAWEC is the viscous coefficient $C_{d}$. Referring to Section 2.5 . case 1 (free decay motion testing) was implemented to estimate $C_{d}$.

Undertaking the free decay test $\left(z_{0}=0.2 \mathrm{~m}\right)$ in the NWT and NSSM, whilst according to the least-squares method described in Eq. (17), $C_{d}$ equal to 1.4 was identified. The results obtained are described in Fig. 8. The displacement amplitude from the NSSM is consistent with the CFD result, whereas a period deviation exists. This arises from the under-predicted total mass of $26.28 \mathrm{~kg}$

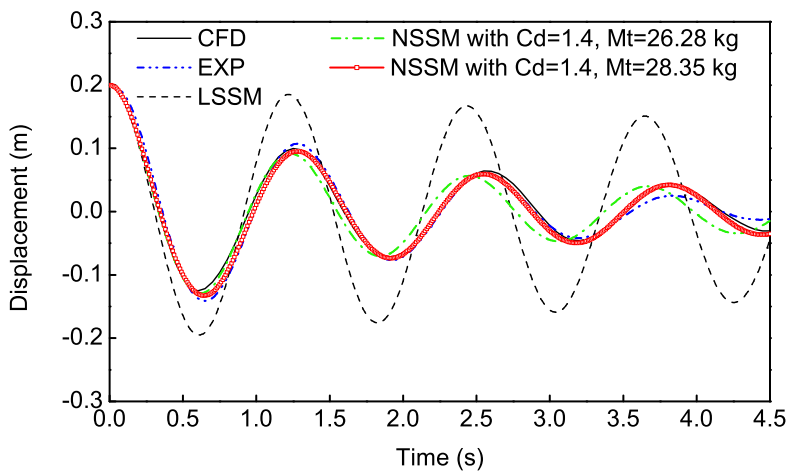

Figure 8: Comparison of the free decay displacements obtained from NSSM, LSSM, CFD and experiment at $z_{0}=0.2 \mathrm{~m}$. 
achieved via the BEM (shown in Fig. 2a). Davidson et al. [23] have also demonstrated the phenomenon that the practical total mass would be different from the linear prediction when the floater oscillation amplitude becomes significant.

To solve this problem, both $C_{d}$ and $M_{t}$ were set as the uncertain parameters in the NSSM. Then repeating the above procedures, $C_{d}$ and $M_{t}$ equalling 1.4 and $28.35 \mathrm{~kg}$, respectively, were obtained. As observed, the achieved result through the NSSM with parameters $C_{d}=1.4, M_{t}=28.35 \mathrm{~kg}$ fits well with CFD output not only in the amplitude evolution but also in the oscillating frequency.

Furthermore, the CFD model and the proposed NSSM were validated by comparing with experimental data shown in Fig. 8 . Clearly, the numerical results of both the CFD and NSSM simulations are in good agreement with the experimental results. The exception is that after $3.5 \mathrm{~s}$ when the buoy motion decays to the equilibrium with low velocity, then the experimental amplitude is slightly lower relative to that from CFD/NSSM. This is mainly due to the friction effect from the roller bearing, which has been discussed in [26].

Fig. 9 shows the normalised displacements against two different $z_{0}(0.2 \mathrm{~m}$ and $0.12 \mathrm{~m}$ ). As expected, the normalised results from the linear model keep identical under different $z_{0}$. Unlike the linear data, the NSSM and CFD results reveal the non-linearity of the free decay response, showing that a higher $z_{0}$ leads to a quicker motion dissipation. Clearly, a higher $z_{0}$ will produce a larger

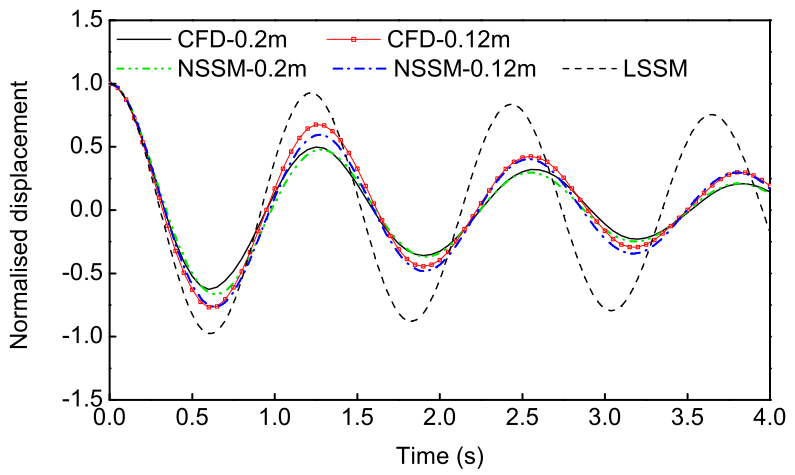

Figure 9: Comparison of the normalised free decay displacements at $z_{0}=0.2$ and $0.12 \mathrm{~m}$. 
relative velocity between the buoy and water, which results in a larger viscous force to hinder the PAWEC movement and consume its kinetic energy. This result concurs with that from the experimental study in 26 .

In 23, a linear parametric hydrodynamic model was identified through CFD data. It shows that the linearised added mass and radiation damping need to be adjusted with varying initial released position so as to properly perform the free decay motion. In comparison, the proposed NSSM in this paper shows improvement by adaptively representing the free decay motion dynamics under different initial position (see Fig. 9).

In summary, it should be noted that compared with the LSSM, the NSSM with $C_{d}=1.4, M_{t}=28.35 \mathrm{~kg}$ performs better in describing the non-linearities associated with the free decay motion. This highlights the potential value of using the designed NSSM in representing wave-PAWEC interactions, which are discussed in the following sections.

\subsection{Viscosity influence on the wave-PAWEC interaction}

According to Section 2.5 case 2 (forced oscillation testing) was conducted to: (i) prove the existence of viscosity in wave-PAWEC interaction; (ii) evaluate the viscosity influence on the PAWEC amplitude and phase responses while interacting with incident wave; (iii) verify the superiority of the NSSM compared with the LSSM in representing the PAWEC hydrodynamics.

\subsubsection{Existence of viscosity in the wave-PAWEC interaction}

Referring to Eq. (14), the viscous force directly depends upon the relative velocity $v_{r}$ between the buoy and the flow, indicating that it is worth observing the $v_{r}$ variations at different wave conditions. Here, the obtained velocity information of PAWEC and the adjacent flow at two representative wave states ( $H$ $=0.073 \mathrm{~m}$ and $\omega=3.12,4.83 \mathrm{rad} / \mathrm{s}$, respectively) are given.

Fig. 10 describes the case that the wave frequency is considerably lower than $\omega_{0}$, equalling $3.12 \mathrm{rad} / \mathrm{s}$. The PAWEC is shown to perform as a "wave follower". Within one oscillation period, the water particles and the buoy reach the peak 

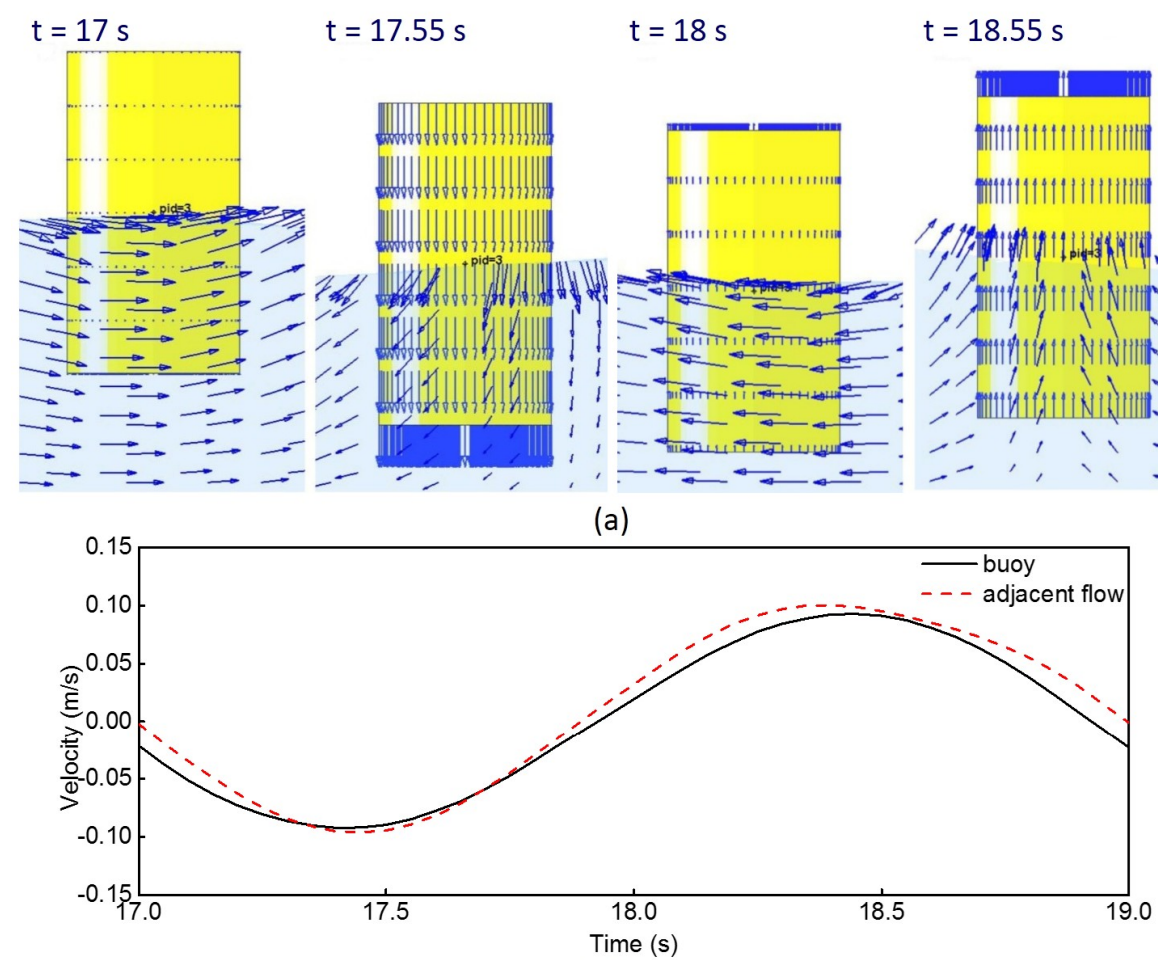

(b)

$t=17.55 \mathrm{~s}$

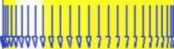

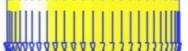

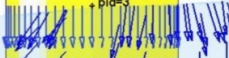


$+\infty$

$\mathrm{t}=18 \mathrm{~s}$

$t=18.55 s$

|III || ||| ||

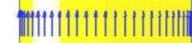

-
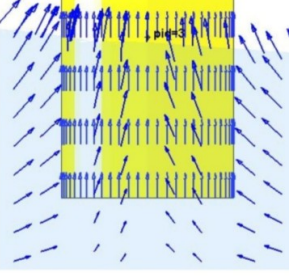

Figure 10: Velocity information of PAWEC and the adjacent flow at $\omega=3.12 \mathrm{rad} / \mathrm{s}, H=$ $0.073 \mathrm{~m}$. (a) Velocity vector distributions. (b) Time series of velocities. The PAWEC shows to track the flow movement synchronously.

\section{.

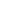




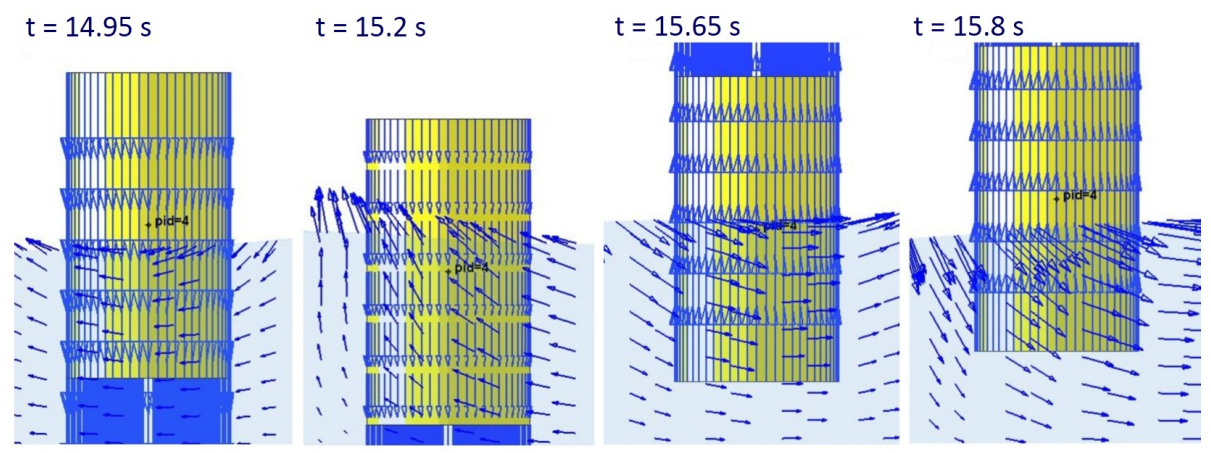

(a)

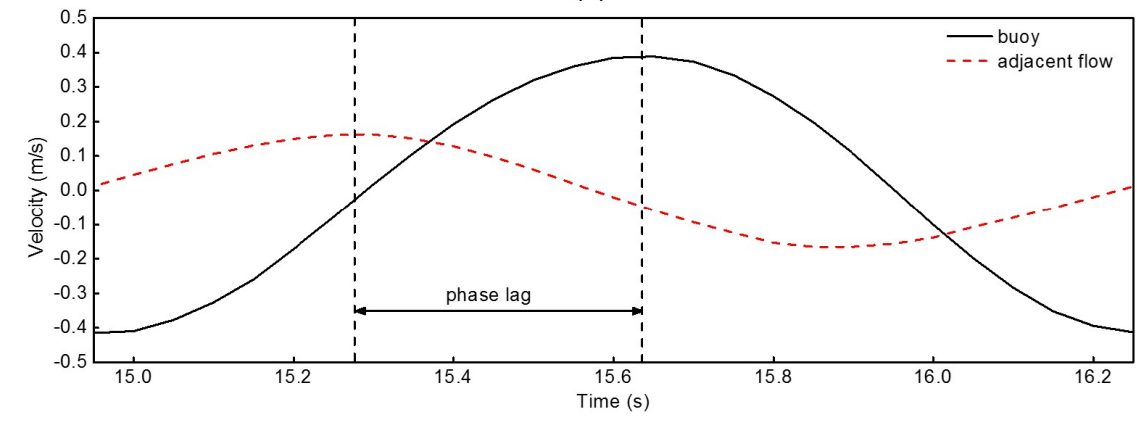

(b)

Figure 11: Velocity information of PAWEC and the adjacent flow at $\omega=4.83 \mathrm{rad} / \mathrm{s}, H=$ $0.073 \mathrm{~m}$. (a) Velocity vector distributions. (b) Time series of velocities. The PAWEC shows to have a clear phase lag relative to the flow.

particles show the opposite trend. Under this situation, the existing phase shift between the PAWEC and the flow would produce non-negligible $v_{r}$. This can generate flow separation and vorticity, causing energy losses. Zang et al. 38 have recorded this phenomenon by experiment and have also suggested the viscous effect on a flat-bottom WEC device.

To summarise, through Figs. 10 and 11 even though the $v_{r}$ is slight when the wave frequency is away from the PAWEC natural frequency, an obvious $v_{r}$ does exist around resonance. This suggests that significant viscous influence may occur in the wave-PAWEC interaction around resonance. This is detailed in the following sections. 


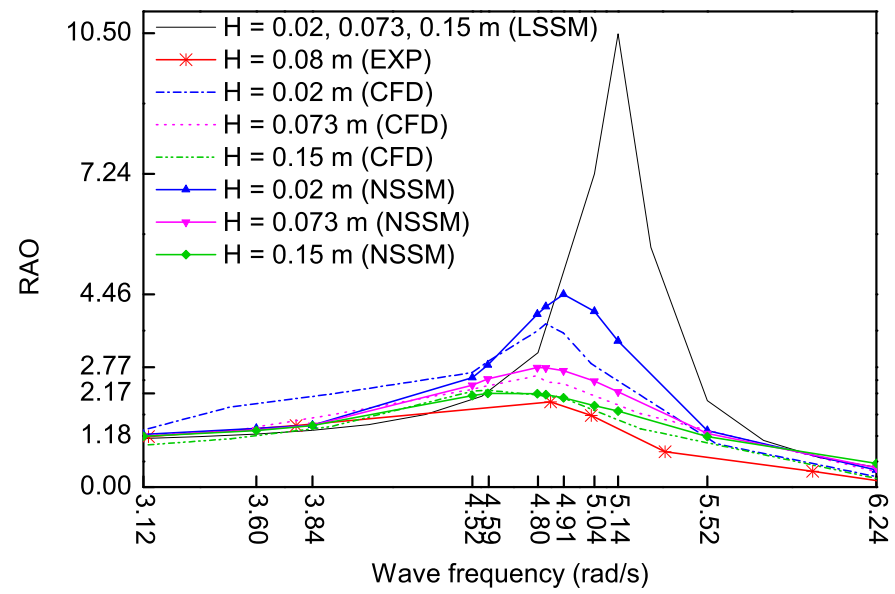

Figure 12: The RAO variation against wave frequency and wave height obtained via LSSM, CFD, NSSM and Experiment.

\subsubsection{Viscosity influence on the PAWEC amplitude response}

Referring to Eqs. (9) and (16), the RAO has two crucial characteristics (maximum value $\mathrm{RAO}_{\max }$ and the wave frequency $\omega_{0}^{\prime}$ occurring $\mathrm{RAO}_{\max }$ ) to predict the efficient wave condition for achieving optimal PAWEC performance.

Fig. 12 plots the RAO against wave frequency at three wave heights. As observed, at relatively low frequencies ( $\omega \leq 3.84 \mathrm{rad} / \mathrm{s})$, the obtained RAO values approximate to 1 using all methods (LSSM, NSSM, CFD and EXP) at different wave heights. The explanation for this can be that under low frequencies the dominant force imposed on the PAWEC is the hydrostatic stiffness term $K z(t)$ (shown in Eq. (1)), which excites the PAWEC to synchronously follow the flow motion with negligible phase lag. This corresponds to the description of velocity information in Fig. 10. Therefore, as expected, with the insignificant viscosity effect at low frequencies, the PAWEC shows no apparent non-linear hydrodynamic performance, and thereby the RAO results are almost independent on the wave height.

However, there are substantial discrepancies among the results from different methods around resonance. First, the $\mathrm{RAO}_{\max }$ is unrealistically over-predicted by LSSM, shown as approximately 5.3 times of that from experiment at 0.08 
${ }_{431} \mathrm{~m}$ wave height (see Table 2). In contrast, the results obtained from NSSM

432 and CFD offer better accordance with the experimental data. The exception is

that both the simulated RAO values appear somewhat higher than the physical wave tank results. These deviations are due to the mechanical friction that exists in the experimental PAWEC system. Second, RAO max and $\omega_{0}^{\prime}$ are constant at different wave heights in the LSSM, whereas showing clear decreases with increasing wave height through NSSM and CFD. These observations could be associated with the different total hydrodynamic damping $B_{h y d}$ for linear and non-linear approaches. Around resonance, with the vanishing reactance in Eqs. (9) and (16), the PAWEC motion is dominated by the damping term $B_{\text {hyd }}$ [16]. Clearly, in the linear model, $B_{h y d}$ (corresponding to the inviscid radiation damp42 ing $B(\omega)$ ) is considerably small and independent of the wave height (see Fig. $2 \mathrm{a}$, which yields the overrated $\mathrm{RAO}_{\max }$, invariant RAO and $\omega_{0}^{\prime}$. Conversely, in 44 the non-linear approaches (NSSM and CFD), the viscosity effect imposed on the PAWEC enhances the total resistance damping. Besides, as described in Eq. (14), a higher wave height would induce a larger relative velocity around reso47 nance (as demonstrated in Fig. 11), which produces a larger viscous damping. 4 Thus both $\mathrm{RAO}_{\max }$ and $\omega_{0}^{\prime}$ show inverse relationships with the wave height.

Similar with the finding in free decay test, the proposed NSSM can adaptively perform free motion dynamics with varying wave height (see Fig. 12). In

\begin{tabular}{|c|c|c|c|c|}
\hline $\mathbf{H}, \mathbf{m}$ & & 0.02 & 0.073 & 0.15 \\
\hline \multirow{2}{*}{ LSSM } & RAO $_{\max }$ & 10.5 & 10.5 & 10.5 \\
\hline & $\omega_{0}^{\prime}, \mathbf{r a d} / \mathrm{s}$ & 5.14 & 5.14 & 5.14 \\
\hline \multirow{2}{*}{ NSSM } & $\mathrm{RAO}_{\max }$ & 4.46 & 2.77 & 2.17 \\
\hline & $\omega_{\mathbf{0}}^{\prime}, \mathbf{r a d} / \mathbf{s}$ & 4.91 & 4.80 & 4.59 \\
\hline \multirow{2}{*}{ CFD } & $\mathrm{RAO}_{\max }$ & 3.78 & 2.58 & 2.24 \\
\hline & $\omega_{\mathbf{0}}^{\prime}, \mathbf{r a d} / \mathbf{s}$ & 4.83 & 4.80 & 4.59 \\
\hline \multirow{2}{*}{ EXP } & $\mathrm{RAO}_{\max }$ & 1 & $1.97^{*}$ & 1 \\
\hline & $\omega_{\mathbf{0}}^{\prime}, \mathbf{r a d} / \mathbf{s}$ & 1 & $4.85^{*}$ & $\backslash$ \\
\hline
\end{tabular}

Table 2: $\mathrm{RAO}_{\max }$ and $\omega_{0}^{\prime}$ at three different wave heights. (Note that $*$ corresponds to the experimental results obtained under wave height of $0.08 \mathrm{~m}$.) 
451

452

contrast, by applying a linearization of the quadratic drag [22, 24], the linearized viscous coefficient has to be adjusted depending on the wave height/velocity amplitudes.

In summary, there is no clear relative motion between the PAWEC and the flow at a low wave frequency. Thus, both linear and non-linear approaches represent the PAWEC amplitude response appropriately. However, due to the indispensable viscosity influence around resonance or at high wave heights, the

NSSM offers a clear improvement in describing the non-linear PAWEC amplitude response against the wave condition. Moreover, it has been observed that the discrepancy between $\omega$ and $\omega_{0}^{\prime}$ increases with increasing wave height. This phenomenon suggests that the optimal condition for power maximization could be dependent on wave height, which is discussed in 3.3 .

\subsubsection{Viscosity influence on the PAWEC phase response}

In addition to the amplitude response, when using regular wave analysis the phase response is another necessary parameter to describe the PAWEC behavior

in the time domain. This section further illustrates the viscosity effect on the phase response.

As expected, Fig. 13 shows the substantial discrepancies of the obtained

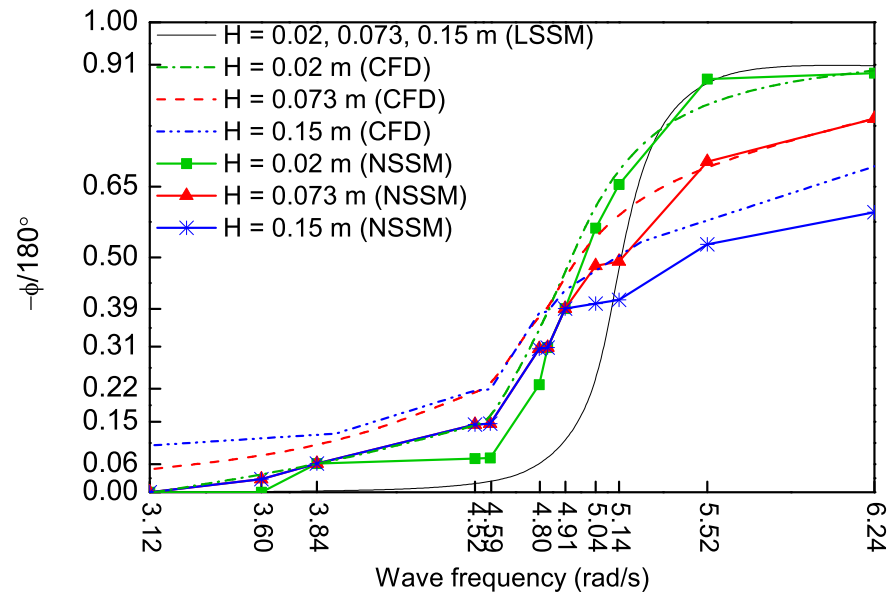

Figure 13: Phase responses at various wave conditions obtained via LSSM, NSSM and CFD. 
phase responses from the linear (LSSM) and the non-linear (NSSM and CFD) approaches, especially at the highest wave height of $0.15 \mathrm{~m}$. By considering viscosity, the NSSM is comparable with the CFD in describing the non-linear PAWEC phase response against the wave height. Moreover, as described in Section 2.1.1 the linear model indicates that resonance $\left(\omega=\omega_{0}=5.14 \mathrm{rad} / \mathrm{s}\right.$, $\mathrm{RAO}_{\max }$ obtained) corresponds to the situation where the floater has approximately $90^{\circ}$ phase lag relative to the flow as shown in Figs. $3 \mathrm{a}$ and 13 . However, Fig. 13 also shows that in the non-linear methods (NSSM and CFD), the obtained phase lag corresponding to the frequency occurring $\mathrm{RAO}_{\max }$ (with reference to $\omega_{0}^{\prime}$ shown in Table 2 is no longer approximate to $90^{\circ}$ at different wave heights. This value shifts further away from $90^{\circ}$ with increasing wave height, as detailed in Fig. 14. This indicates that in contrast to the linear theory, in practice, the optimal criteria: $\mathrm{RAO}_{\max }$ and nearly $90^{\circ}$ phase lag of the PAWEC motion relative to the flow cannot be achieved at resonance frequency $\omega_{0}$. In other words, the LSSM loses effectiveness in representing the PAWEC hydrodynamics in the cases of large oscillations.

To further demonstrate the improvement of NSSM in describing the PAWEC hydrodynamic behavior, two illustrative examples in time domain are discussed. Fig. 15 shows the velocity time evolutions of the PAWEC and the flow at $\omega$

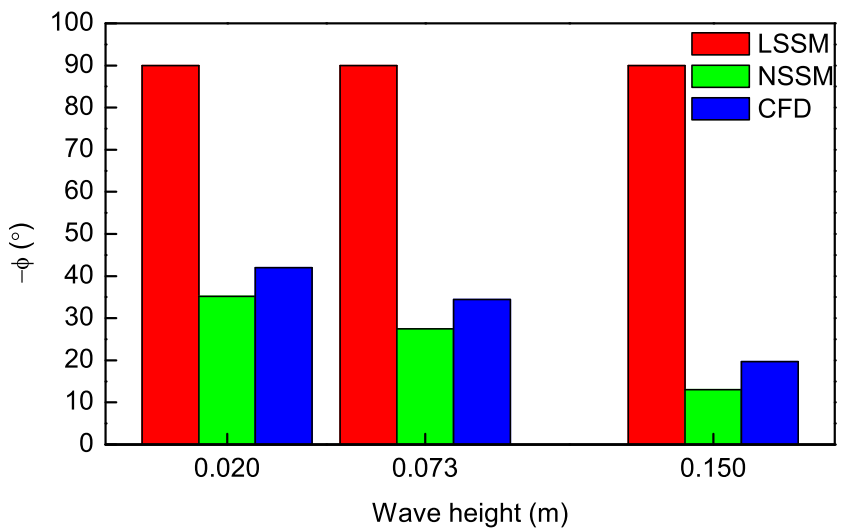

Figure 14: The PAWEC motion phase lag (relative to the flow motion) against wave height when $\mathrm{RAO}_{\max }$ is achieved. 


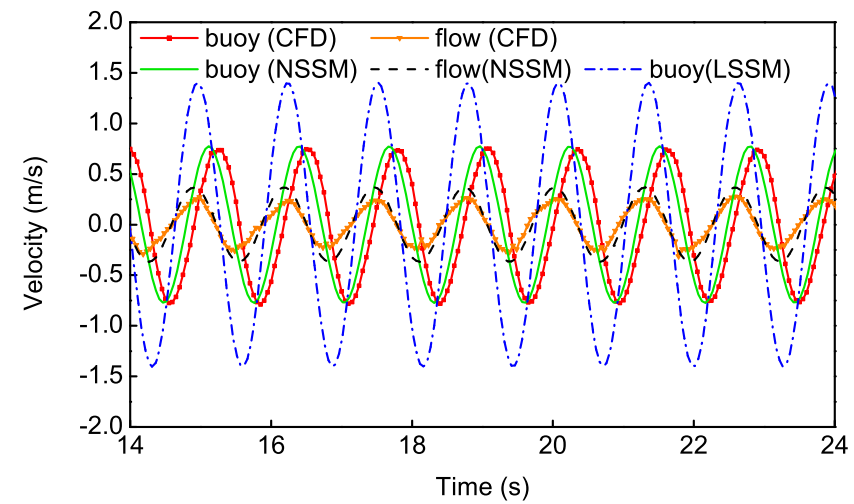

Figure 15: Velocity time series of the PAWEC and the flow at $\omega=4.91 \mathrm{rad} / \mathrm{s}, H=0.15 \mathrm{~m}$.

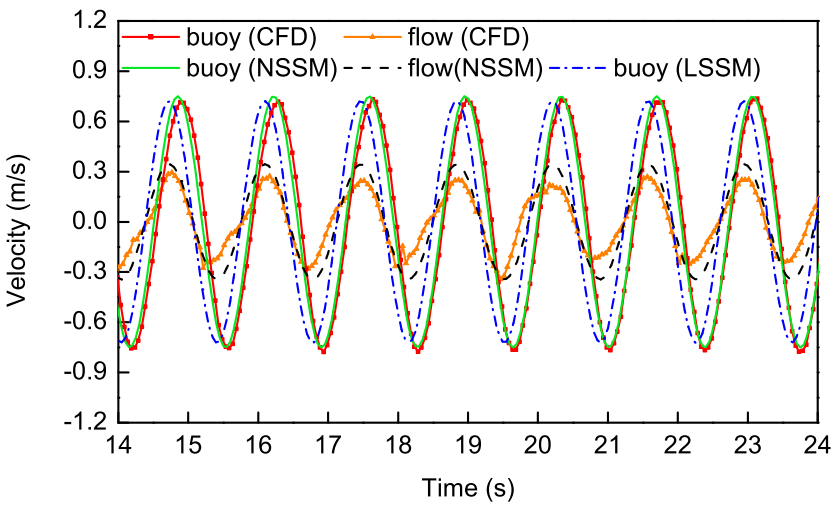

Figure 16: Velocity time series of the PAWEC and the flow at $\omega=4.59 \mathrm{rad} / \mathrm{s}, H=0.15 \mathrm{~m}$.

$488=4.91 \mathrm{rad} / \mathrm{s}, H=0.15 \mathrm{~m}$. Clearly, the PAWEC velocity achieved via the LSSM deviates from the CFD result severely, with a $80^{\circ}$ phase lead and twice amplitude. Furthermore, when $\omega=4.59 \mathrm{rad} / \mathrm{s}, H=0.15 \mathrm{~m}$ (shown in Fig. 16), even if the PAWEC velocity magnitude through the LSSM fits well with the

492 CFD data (associated with the similar RAO values predicted at this frequency 493 shown in Fig. 12, a $48.6^{\circ}$ phase lead still exists relative to the CFD output.

494 In contrast, the NSSM is shown to perform better in representing not only the 495 amplitude response but also the phase response for the designed PAWEC. 


\subsection{Power conversion efficiency of the designed PAWEC}

Through the observations in Sections 3.1 and 3.2 , the viscosity affects the designed PAWEC to perform non-linearities at different wave heights. This implies that the practical power conversion efficiencies of the PAWEC may deviate from the predicted results through the linear model. Thus referring to Section 2.5 case 3 (power conversion efficiency testing) was conducted to evaluate the viscosity influence on the PAWEC power conversion efficiency.

Fig 17a shows the power conversion efficiency variation against the dimen-

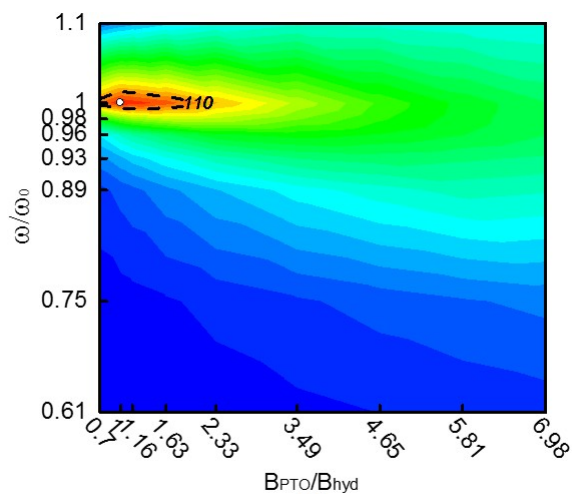

(a)

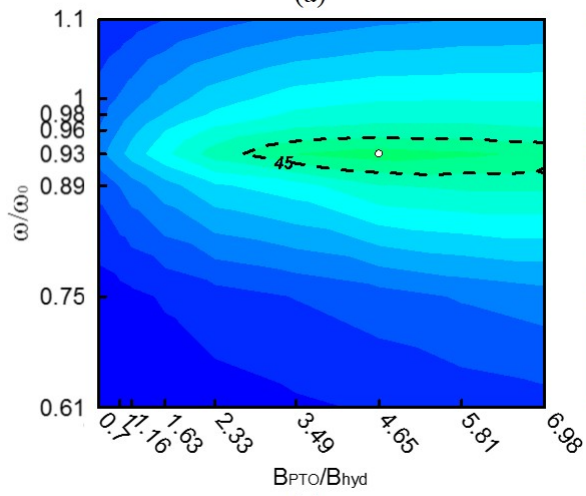

(c)

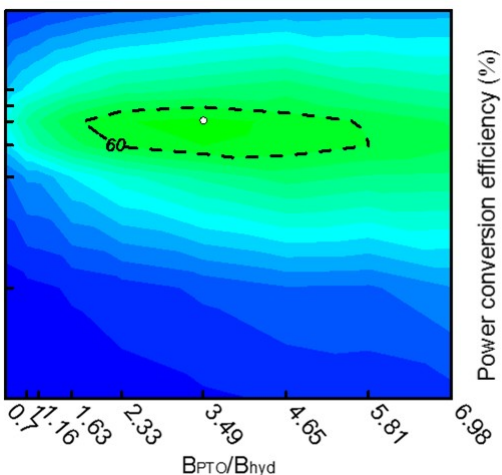

(b)

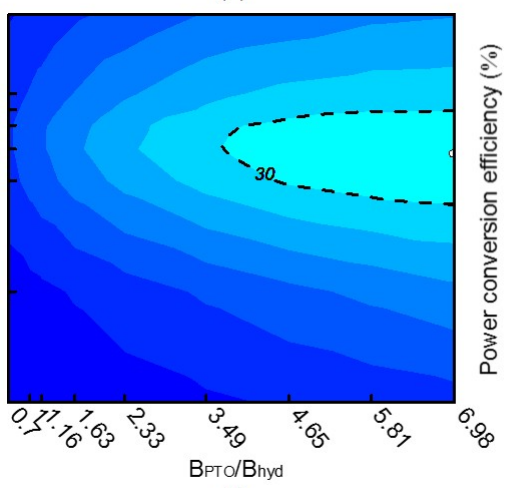

(d)

Figure 17: Power conversion efficiency against the dimensionless PTO damping coefficient and wave frequency. Note that: $B_{h y d}=4.3 \mathrm{Ns} / \mathrm{m}$; the white point represents the maximum efficiency. (a) At $H=0.073 \mathrm{~m}$ through LSSM, maximum efficiency of $125 \%$ (b) At $H=0.02 \mathrm{~m}$ through NSSM, maximum efficiency of $66.6 \%$.(c) At $H=0.073 \mathrm{~m}$ through NSSM, maximum efficiency of $52.5 \%$. (d) At $H=0.15 \mathrm{~m}$ through NSSM, maximum efficiency of $33.5 \%$. 
sionless PTO damping coefficient and wave frequency at a wave height of 0.073 $m$ through the LSSM. As expected, the floater achieves the optimal power conversion efficiency of $125 \%$ at $\omega / \omega_{0}=1$ and $B_{P T O} / B_{h y d}=1$ (for the designed PAWEC, $B_{h y d}=4.3 \mathrm{Ns} / \mathrm{m}$ is achieved at resonance, shown in Fig. 2a). Besides, the efficiency value is affected by the wave frequency enormously, showing a sharp decrease with the wave frequency away from the PAWEC natural frequency, especially at low PTO damping coefficients. Additionally, the PTO damping coefficient and the wave frequency are dependent on each other. Firstly, around resonance (inside the dash line), the power conversion efficiency declines gradually while the PTO damping value departing from $B_{h y d}$. Conversely, a larger PTO damping value could produce a higher conversion efficiency when the wave frequency is out of the resonance zone (outside the dash line). These could be associated with the amplitude responses predicted through the linear model that overrated/abruptly decreased motion responses in/away the resonance zone, respectively(see Fig. $3 \mathrm{a}$ or 12 .

With the consideration of viscosity, the NSSM shows different power conversion efficiency performance see Fig. 17-d). When the wave height grows, the optimal damping increases, while the optimal wave frequency decreases. This indicates that the parameters corresponding to the maximum efficiency shift away from their theoretical optimal values based on the linear theory. Similar findings can be found in the CFD and experimental studies reported in [39, 40]. This may be caused by two effects: (i) in the NSSM, the viscous-damping coefficient has been involved in $B_{h y d}$, which contributes to the $B_{P T O}$ variation with respect to different wave conditions. At small wave heights, viscous influence is negligible. Hence, $B_{h y d}$ could be approximated to be linear leading to the optimal condition close to the theoretical value. However, at high wave heights, due to the indispensable viscosity influence, $B_{h y d}$ significantly increases which requires a higher optimal PTO damping to reduce energy loss. (ii) It is well known that the optimal conversion efficiency is dependent on the largest amplitude response of the PAWEC. As described in Section 3.2.2, under a higher wave height, the wave frequency at which the maximum PAWEC amplitude response 
535

occur shifts to the lower frequency. Therefore, the optimal wave frequency for the maximum power conversion efficiency is shown to be lower when the wave height grows.

For the wave height of $0.073 \mathrm{~m}$, the NSSM predicts the maximum power conversion efficiency of $52.5 \%$ for the designed PAWEC, which is more reasonable compared with the efficiency of $125 \%$ estimated through the linear model. In addition, comparing the power conversion efficiency against wave height shown in Fig. 17 - $d$, it can be found that the growth of the wave height yields the decrease of efficiency.

In practice, we suppose that the optimal PAWEC operation range is a decrement of $10 \%$ power conversion efficiency relative to the maximum value. Through the NSSM, the range for the efficient power conversion efficiency seems to be expanded compared with the narrow optimal range predicted in the linear theory. For the wave conditions and PTO damping coefficients studied in this work, the optimal condition for the designed PAWEC varies in the range: 10.75 $\mathrm{Ns} / \mathrm{m}<B_{P T O}<24.7 \mathrm{Ns} / \mathrm{m}$ together with $4.7 \mathrm{rad} / \mathrm{s}<\omega<5.0 \mathrm{rad} / \mathrm{s}$.

\section{Conclusions}

In this work, the viscosity influence on the hydrodynamic performance and power conversion efficiency of the designed $1 / 50$ scale vertical oscillating PAWEC was investigated by comparing results obtained through LSSM and NSSM with CFD and experimental data. Some conclusions are drawn as follows:

- The viscous coefficient and total mass of 1.4 and $28.35 \mathrm{~kg}$ for the designed PAWEC have been predicted by comparing the free decay test result from the NSSM with the CFD output. As a result, the proposed NSSM fits well with the CFD and experiment in describing the non-linearity of the PAWEC free decay motion (see Fig. 9).

- Using forced oscillation testing, the conventional LSSM is shown to lose effectiveness in describing both the PAWEC amplitude and phase responses. 
Conversely, the proposed NSSM is comparable with the CFD and experiment in representing the non-linear hydrodynamic behaviors at different wave heights. The results suggest that the conventional optimal performance criteria at the resonance frequency such as maximum oscillation and approximately $90^{\circ}$ phase lag between PAWEC and regular wave motion are not valid as wave height increases (see Figs. 12 and 13 . With the viscosity influence, the PAWEC RAO and phase responses would have different performances under different wave heights.

- Based on the conventional linear modeling approach, an unreasonable power conversion efficiency of $125 \%$ can be found at a wave height of $0.073 \mathrm{~m}$ (shown in Fig. 17a). Additionally, the wave frequency is seen to be the most crucial factor affecting the conversion efficiency. Of next importance in this context is the PTO damping coefficient using the linear theory. Nevertheless, according to the NSSM, the maximum efficiency of $52.5 \%$ was obtained at a wave height of $0.073 \mathrm{~m}$. In addition to wave frequency and PTO damping, the power conversion efficiency is also affected by wave height. Moreover, the optimal condition for the maximum efficiency is no longer consistent compared with the linear theory, which is influenced by the wave height. A higher wave height could induce the optimal conditions corresponding to a higher PTO damping and a lower wave frequency (see Fig. 17-d).

To summarise, the work shows that for the designed 1/50 scale PAWEC, the LSSM fails to accurately predict the hydrodynamic performance and power conversion efficiency, especially around resonance or at high wave heights. In contrast, when considering an appropriate quadratic viscosity term the NSSM shows better potential for reproducing the non-linear hydrodynamic performance under variable wave conditions (wave height and wave frequency). This highlights the non-negligible viscosity influence on the PAWEC hydrodynamics. In future work, it is expected to apply the designed NSSM as a control plant for achieving optimal PAWEC performance. Furthermore, since viscosity could dissipate 
the PAWEC mechanical energy, methods to reduce viscous influence have been ongoing, for example based on the inclusion of geometry optimization in the design of PAWEC systems 41. Finally, using a combination of geometric optimization and non-linear modeling for more complex WEC device structures, it is expected that the results of this paper can form a valuable basis for PTO and advanced control within the power maximization framework.

\section{Acknowledgements}

Siya Jin and Bingyong Guo wish to acknowledge the China Scholarship Council (CSC) and the University of Hull for joint scholarships. Great thanks are expressed to Dr Stuart Mclelland, Professor Dan Parsons and Mr Brendan Murphy of the School of Geography, Environment \& Earth Sciences for the management and support of the Hull University flume tank.

\section{References}

[1] I. Glendenning, Ocean wave power, Appl. Energy 3 (1977) 197-222.

[2] J. Cruz, Ocean wave energy: current status and future prespectives, Springer Science \& Business Media, 2007.

[3] S. Salter, D. Jeffery, J. Taylor, The architecture of nodding duck wave power generators, The Naval Architect 1 (1976) 21-24.

[4] M. Eriksson, J. Isberg, M. Leijon, Hydrodynamic modelling of a direct drive wave energy converter, Int. J. Eng. Sci. 43 (2005) 1377-1387.

[5] F. d. O. Antonio, Wave energy utilization: A review of the technologies, Renew. Sust. Energy Rev. 14 (2010) 899-918.

[6] V. DelliColli, P. Cancelliere, F. Marignetti, R. DiStefano, M. Scarano, A tubular-generator drive for wave energy conversion, IEEE Trans. Ind. Electron. 53 (2006) 1152-1159. 
[7] J. Hals, J. Falnes, T. Moan, Constrained optimal control of a heaving buoy wave-energy converter, J. Offshore Mech. Arct. Eng. 133 (2011) 011401.

[8] J. Goggins, W. Finnegan, Shape optimisation of floating wave energy converters for a specified wave energy spectrum, Renew. Energy 71 (2014) 208-220.

[9] D. Son, R. W. Yeung, Optimizing ocean-wave energy extraction of a dual coaxial-cylinder wec using nonlinear model predictive control, Appl. Energy 187 (2017) 746-757.

[10] Y. Li, Y.-H. Yu, A synthesis of numerical methods for modeling wave energy converter-point absorbers, Renew. Sust. Energy Rev. 16 (2012) 4352-4364.

[11] Z. Yu, J. Falnes, State-space modelling of a vertical cylinder in heave, Appl. Ocean Res. 17 (1995) 265-275.

[12] R. Taghipour, T. Perez, T. Moan, Hybrid frequency-time domain models for dynamic response analysis of marine structures, Ocean Eng. 35 (2008) 685-705.

[13] A. Babarit, A. H. Clément, Optimal latching control of a wave energy device in regular and irregular waves, Appl. Ocean Res. 28 (2006) 77-91.

[14] T. K. Brekken, On model predictive control for a point absorber wave energy converter, in: Power Tech., IEEE, 2011, pp. 1-8.

[15] G. De Backer, Hydrodynamic design optimization of wave energy converters consisting of heaving point absorbers, Department of Civil Engineering, Ghent University: Ghent, Belgium (2009).

[16] J. Journée, W. Massie, Offshore hydromechanics, TU Delft, 2000.

[17] J. Falnes, Ocean waves and oscillating systems: linear interactions including wave-energy extraction, Cambridge university press, 2002. 
[18] M. Vantorre, R. Banasiak, R. Verhoeven, Modelling of hydraulic performance and wave energy extraction by a point absorber in heave, Appl. Ocean Res. 26 (2004) 61-72.

[19] M. Eriksson, R. Waters, O. Svensson, J. Isberg, M. Leijon, Wave power absorption: Experiments in open sea and simulation, J. Appl. Phys. 102 (2007) 084910.

[20] Y.-H. Yu, Y. Li, Reynolds-averaged navier-stokes simulation of the heave performance of a two-body floating-point absorber wave energy system, Computers \& Fluids 73 (2013) 104-114.

[21] Y. Wei, A. Rafiee, A. Henry, F. Dias, Wave interaction with an oscillating wave surge converter, part i: Viscous effects, Ocean Eng. 104 (2015) 185203.

[22] D. Son, V. Belissen, R. W. Yeung, Performance validation and optimization of a dual coaxial-cylinder ocean-wave energy extractor, Renew. Energy 92 (2016) 192-201.

[23] J. Davidson, S. Giorgi, J. V. Ringwood, Linear parametric hydrodynamic models for ocean wave energy converters identified from numerical wave tank experiments, Ocean Eng. 103 (2015) 31-39.

[24] S. J. Beatty, M. Hall, B. J. Buckham, P. Wild, B. Bocking, Experimental and numerical comparisons of self-reacting point absorber wave energy converters in regular waves, Ocean Eng. 104 (2015) 370-386.

[25] M. A. Bhinder, A. Babarit, L. Gentaz, P. Ferrant, Potential time domain model with viscous correction and cfd analysis of a generic surging floating wave energy converter, Int. J. Mar. Energy 10 (2015) 70-96.

[26] B. Guo, R. Patton, S. Jin, J. Gilbert, D. Parsons, Nonlinear modeling and verification of a heaving point absorber for wave energy conversion, IEEE Trans. Sustain. Energy 9 (2018) 453-461. 
[27] B. Guo, R. Patton, M. Abdelrahman, J. Lan, A continuous control approach to point absorber wave energy conversion, in: 11th UKACC, IEEE, 2016, pp. 1-6.

[28] M. Abdelrahman, R. Patton, B. Guo, J. Lan, Estimation of wave excitation force for wave energy converters, in: 3rd SysTol, IEEE, 2016, pp. 654-659.

[29] K. Budar, J. Falnes, A resonant point absorber of ocean-wave power, Nature 256 (1975) 478-479.

[30] W. Cummins, The impulse response function and ship motions, Technical Report, DTIC Document, 1962.

[31] T. F. Ogilvie, Recent progress toward the understanding and prediction of ship motions, in: 5th Symposium on naval hydrodynamics, volume 1, Bergen, Norway, 1964, pp. 2-5.

[32] M. E. McCormick, Ocean wave energy conversion, Courier Corporation, 2013.

[33] J. Morison, J. Johnson, S. Schaaf, et al., The force exerted by surface waves on piles, JPT 2 (1950) 149-154.

[34] J. O. Hallquist, Ls-dyna theory manual, LSTC 3 (2006) 25-31.

[35] R. G. Dean, R. A. Dalrymple, Water wave mechanics for engineers and scientists, volume 2, World Scientific Publishing Co Inc, 1991.

[36] F. Ursell, R. G. Dean, Y. Yu, Forced small-amplitude water waves: a comparison of theory and experiment, J. Fluid Mech. 7 (1960) 33-52.

[37] M. CREWE, The national meteorological library and archives, State librarian 38 (1990) 37-39.

[38] Z. Zang, Q. Zhang, Y. Qi, X. Fu, Hydrodynamic responses and efficiency analyses of a heaving-buoy wave energy converter with pto damping in regular and irregular waves, Renew. Energy 116 (2018) 527-542. 
696 [39] J. Davis, COULOMB, Wave energy absorption by the bristol cylinder-

697 linear and non-linear effects., Proc Inst Civil Eng 89 (1990) 317-340.

[40] M. Anbarsooz, M. Passandideh-Fard, M. Moghiman, Numerical simulation of a submerged cylindrical wave energy converter, Renew. Energy 64 (2014) $132-143$.

[41] S. Jin, R. Patton, Geometry influence on hydrodynamic response of a heaving point absorber wave energy converter, in: 12th EWTEC, 2017. 